\title{
DiverseK: integrating paleoecology, traditional knowledge and stakeholders
}

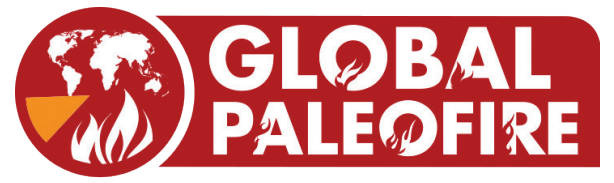

\author{
Daniele Colombaroli', J. Mistry², A. Milner', B. Vannière ${ }^{3}$, C. Adolf4, D. Hawthorne ${ }^{5}$ and the Global Paleofire \\ Working Group (GPWG2)
}

\section{Egham, UK, 4-7 September 2018}

Integration of ecosystem science and applied research in ecosystem management is a high priority and key challenge for the science-policy interface, as recently highlighted by the Intergovernmental Science-Policy Platform on Biodiversity and Ecosystem Services (IPBES 2018). A more direct involvement of stakeholders and poli cymakers into the research agenda requires new approaches for knowledge transfer from the academic to the stakeholder community, as also emphasized during previous workshops organized by the Global Paleofire Working Group GPWG2 (Blarquez et al. 2018; Courtney-Mustaphi et al., this issue).

Thirty participants from 15 countries (Fig. 1) met at Royal Holloway University of London, UK, to discuss ongoing challenges on biodiversity conservation and fire policy, considering three approaches: (a) long-term ecology - informing on ecosystem responses to environmental change across regions and timescales (paleoecology-informed conservation); (b) local, traditional, and indigenousknowledge systems on fire management that maintain biodiversity (community-owned and -driven conservation); and (c) conservation challenges and agendas defined by stakeholders and policymakers (stakeholder driven research). The combination of long term ecology with traditional knowledge represents a novel and alternative approach to promote a more sustainable management practice of present ecosystems under current threats, and fosters the dialogue between the different disciplines.

\section{Before the workshop, conservation and} fire-management evidence priorities were identified together with the UK Government Department for Environment, Food and Rural Affairs, and included: (1) the impact of changing climate and land use on fire regimes; (2) ecosystem recovery after fires of different severity; (3) the optimum fire regimes needed to achieve management objectives for biodiversity conservation; and (4) the effects of prescribed burning (and other land-management practices) on wildfires. The participants discussed examples of fire policies and relative impacts based on their regional expertise and on a field discussion in Chobham Common (Fig. 1). Different sub-groups then compiled evidence-based case studies on past ecosystem legacies, the role of local ecological knowledge in maintaining landscapes (Mistry and Berardi 2016), post-fire ecosystem dynamics, and burning conditions that optimize biodiversity
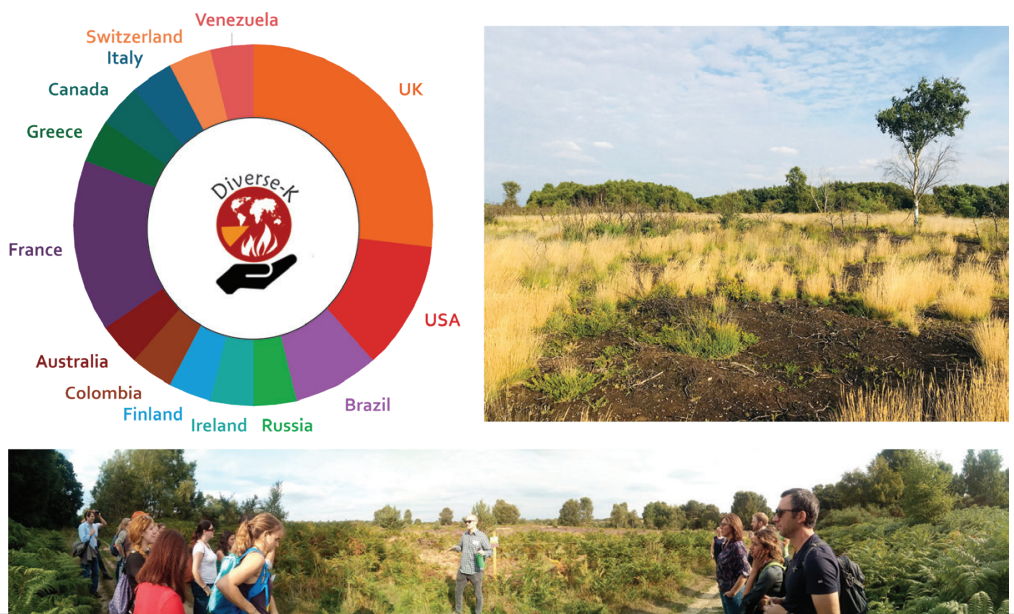

Figure 1: (Left) Distribution of the participants' home countries. (Right and bottom) Guided discussion on fire risk and lowland heath-conservation priorities at Chobham Common, the largest National Nature Reserve in South East England. Photos: A. Milner and D. Colombaroli.

across ecosystems (Colombaroli et al. 2013). This approach also highlighted potential conflicts in conservation targets for specific regions (natural vs cultural; e.g. Jackson and Hobbs 2009), and the need to anticipate new challenges in a future warmer world (Fischer et al. 2018). The participants produced a first draft of a policy brief summarizing best practices for sustainable ecosystem management, including how transdisciplinary knowledge can inform fire management and policy, and highlighted future priorities to foster effective science-policy knowledge exchange in this region. In addition, a group of early-career researchers focused on the compilation of a systematic review protocol to summarize the available evidence on the effects of climate change and land use on fire regimes.

Adapting fire policy to more sustainable practices for specific regions requires approaches that focus primarily on the most urgent challenges set by policymakers (policy-driven research), in co-production with diverse knowledge and expertise (here paleoecology, cultural geography and policymakers). This dialogue can lead to the implementation of decision tools for policymakers, and further promote the integration of long-term ecology in more applied science (Willis and Birks 2006). The Global Paleofire Working Group, through the Diverse Knowledge framework (DiverseK), will continue to build a strong network of researchers, land managers, practitioners, and policymakers to tackle ongoing challenges in ecosystem management and biodiversity conservation

\section{ACKNOWLEDGEMENTS}

Funding has been provided by PAGES, the Quaternary Research Association (QRA), and Chrono-environnement at Université Bourgogne Franche-Comté. The full meeting program is available at gpwg.paleofire.org/event/focus-group-3-workshop Iondon-2018. Additional material is on our Twitter ac count (@diverse_K). The motivation for this workshop was inspired by Cathy Whitlock, the GPWG community (gpwg.paleofire.org) and previous PAGES meetings in 2012 by K.J. Willis and E. Jeffers in Oxford, UK, (PAGES Focus 4 Biodiversity Theme Workshop, pastglobalchanges.org/calendar/127-pages/1006) and in 2017 in Bern, Switzerland, by H. Fischer, K. Meissner and A. Mix (PAGES Warmer Worlds Integrative Activity workshop, pastglobalchanges.org/calendar/127-pages/1653).

AFFILIATIONS

'Centre for Quaternary Research, Department of Geography, Royal Holloway University of London, UK Department of Geography, Royal Holloway University of London, UK

UMR Chrono-environnement (CNRS), Universite Bourgogne Franche-Comté, Besançon, France Department of Zoology, University of Oxford, UK ${ }^{5} \mathrm{AOC}$ Archaeology Group, Edinburgh, UK

\section{CONTACT}

Daniele Colombaroli: daniele.colombaroli@rhul.ac.uk

REFERENCES

Blarquez et al. (2018) PAGES Mag 26(1): 40

Colombaroli D et al. (2013) Divers Distributions 19: $157-170$

Fischer H et al. (2018) Nat Geo 11: 474-485

IPBES (2018) Plenary of the Intergovernmental SciencePolicy Platform on Biodiversity and Ecosystem

Services Sixth session, $1151 \mathrm{pp}$

Jackson S, Hobbs RJ (2009) Science 325: 567-569 Mistry J, Berardi A (2016) Science 352: 1274-1275 Willis KJ, Birks HJB (2006) Science 314: 1261-1265 